\title{
Ozone and its use in periodontal treatment
}

\author{
Dimitrios Iliadis ${ }^{1}$, Brian J. Millar ${ }^{2 *}$ \\ ${ }^{1}$ Prosthodontic Practice, Southchurch Family Dental Centre, Nottingham, UK \\ ${ }^{2}$ Primary Care Dentistry, King's College London Dental Institute at Guy's, King's College \& St. Thomas' Hospitals, London, UK \\ Email: brian.millar@kcl.ac.uk
}

Received 11 February 2013; revised 25 April 2013; accepted 8 May 2013

Copyright (C) 2013 Dimitrios Iliadis, Brian J. Millar. This is an open access article distributed under the Creative Commons Attribution License, which permits unrestricted use, distribution, and reproduction in any medium, provided the original work is properly cited.

\begin{abstract}
Objectives: To evaluate the effects of ozone in periodontal treatment in dental practice. Methods: An evaluation of the current state of knowledge regarding the application of ozone in periodontal treatment revealed limited available literature. Therefore an audit was conducted in dental practice in order to evaluate the effects of ozone in periodontal treatment. Twentyfive patients were treated with gaseous ozone after having had failed conventional periodontal treatment. BPE scores and the six deepest pockets were measured in each patient before and after the use of ozone. Results: From the initial number of twenty-nine patients selected, twenty-five patients attended both follow up appointments. Based on BPE scores, twenty of the patients have overall improvement while five of the patients continued to have deterioration. Eight patients had an improvement in depths of periodontal pockets by three millimetres, sixteen patients had improvement by one to two millimetres and one patient did not improve. The depth of pockets after the use of ozone decreased significantly $(P<0.001)$. Conclusion: The audit revealed that gaseous ozone significantly $(P$ $<0.001)$ reduced the depth of pockets in patients with periodontal disease. The positive results encourage further investigation in the subject.
\end{abstract}

Keywords: Ozone; Periodontal; Audit

\section{INTRODUCTION}

Ozone was discovered by Schonbein in 1840 and has been used widely in industry [1]. Schonbein reported that the electrolysis of water produced an odour at the positive electrode [2]. He named that ozone from the ancient Greeks who also observed this strong odour after electric storms and they called it "ozein" ("to have a smell").

\footnotetext{
*Corresponding author.
}

Ozone is a triatomic molecule and exists as colourless gas with a pungent odour at room temperature, detectable at concentrations as low as 0.02 to $0.05 \mathrm{ppm}$ (by volume) [3]. It is a highly corrosive, toxic and a powerful oxidant. Ozone exists in the atmosphere with the highest levels are in stratosphere in the region known as the ozone layer between 10 and $50 \mathrm{~km}$ above the sea level. In nature, ozone is also commonly found as a result of lightning strikes during thunderstorms and waterfalls [2]. Ozone absorbs dangerous B and $\mathrm{C}$ ultraviolet radiations making it very useful, yet it can be also very toxic for the pulmonary tract especially when it mixes with carbon monoxide (CO), $\mathrm{N}_{2} \mathrm{O}$ and traces of acids as it occurs in $\operatorname{smog}[1]$.

In dentistry ozone has been recognised for its antimicrobial effect and it can be used as a useful disinfectant in clinical applications [4,5]. It is a part of the evolving minimally invasive dentistry theme (MI) and it's aim of preserving the original tissues where possible. It is claimed that ozone promotes haemostasis, enhances local oxygen supply and inhibits bacterial proliferation [1]. Although there are some promising studies, ozone has not been proven superior to other clinical approaches [6]. There is still need for more scientific data on the subject, as clinical evidence for application of ozone in dentistry is not extensive [4]. Furthermore, there is little evidence for the use of ozone in periodontal treatment and there is a need for more studies in this particular field [7-10].

It is known that ozone can kill bacteria by rupturing their cell membranes within a few seconds. In medicine and dentistry, ozone is used as a powerful sterilizing agent either in the gaseous or aqueous phase, as it successfully kills bacteria, fungi and viruses. Ozone has been found to have a bactericidal effect, particularly in staphylococcal, streptococcal and other infections. Recent research showed that exposure of carious dentine specimens to ozone reduced the levels of pathogenic microorganisms in these samples [4]. Ozone can be used for sterilisation of heat sensitive materials including medical 
devices and narrow lumen devices [11]. Some researchers [12] found that even soaking toothbrushes contaminated with oral microorganisms in ozonated water has good disinfectant results. There are currently two ozone generators used in clinical dental applications and both utilise corona discharge (HealOzone by KaVo and Ozotop by TTT, see Figure 1).

Ozone has powerful microbicidal properties, however, this action not only affects micro-organisms but also all the other living systems. Ozone's concentration in the blood is very important and high levels can be very cytotoxic producing even haemolysis. Baysan and Lynch [13] have listed the potential advantages and adverse reaction of ozone in clinical use.

Periodontitis is chronic gingivitis with associated loss of attachment. The development and course of periodontitis appears to be dependent upon specific inherited, behavioural or environmental conditions - so called risk factors. These risk factors are biologically linked to the disease. There are certain risk determinants (genetics, socioeconomic status and gender) that cannot be modified. In periodontitis treatment is based initially on the supragingival calculus removal and possibly root planning combined with oral hygiene instructions. However, the clinical signs of illness (including the presence of microorganisms) can still be found after mechanical debridement, so chemomechanical treatment approach has been suggested as being effective. Ozone may be effective because of its antimicrobial effect.

A literature review revealed little clinical data on the use of gaseous ozone in periodontal disease and conflicting opinions on its benefit [7-10]. Therefore the authors decided to review outcomes from patients under treatment. Recent papers have reported a greater reduction in plaque index, gingival index and bleeding index following the use of ozone irrigation compared to the use of chlorhexidine [14]. Huth et al. [15] similarly showed significant results with gaseous and aqueous ozone and concluded that they merit further investigation. A study by

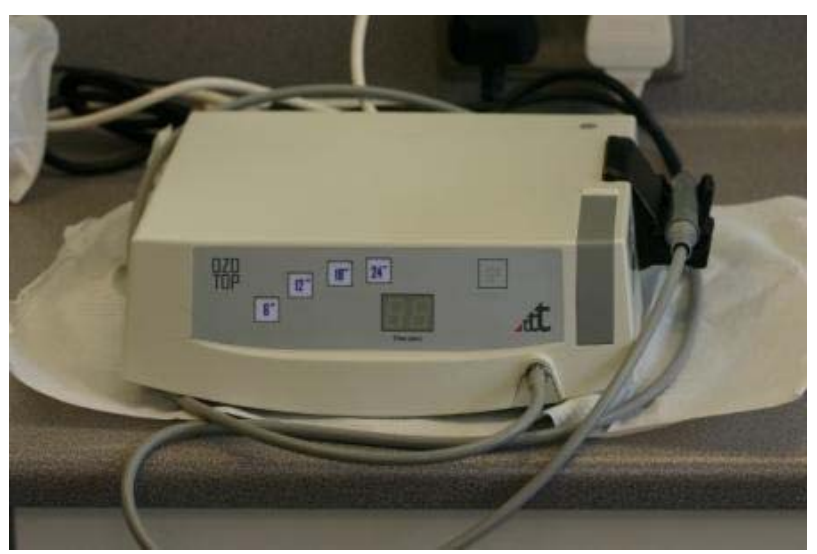

Figure 1. The ozone generator used by the authors.
Hauser et al. [16] investigated the use of gaseous ozone on bacteria adhering to implant surfaces and showed a selective reducation in bacteria, concluding that gaseous ozone may have a role in treatment of peri-implantitis.

The aim of the audit was to assess the effect of ozone which is being currently used as an adjunctive treatment in the management of periodontal disease in a UK General Dental Practice.

\section{MATERIALS AND METHODS}

An audit was been undertaken to answer the clinical question whether the ozone makes a difference in the treatment of periodontal disease.

\subsection{Strategy and Subjects}

From December 2007 all the patients who presented with periodontal disease in one of the author's practice (DI), were treated with conventional periodontal treatment. This includes oral hygiene instruction and follow-up, supra and subgingival scaling and a Basic Periodontal Examination, (BPE). The BPE, as defined by the British Society of Periodontology [17], requires that the periodontal tissue should be examined with a standardised periodontal probe using light pressure to examine the tissue for bleeding, plaque retentive factors and pocket depth. A code 0 - 4 is then determined as follows:

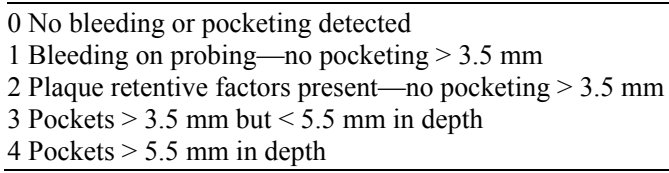

The patients constituted the regular, everyday patients' list. Twenty-nine patients (16 female, 13 male) aged between thirty-two and fifty-four, who failed to respond well to the conventional periodontal treatment were offered further treatment with ozone. The treatment with ozone, including potential risks and benefits, was fully explained. This was the normal treatment procedure within the practice. An audit was carried out to review the outcomes and assess the benefits of this approach as a guide to designing future treatment strategy in the practice.

Five patients did not receive ozone therapy, so could not be included in the audit, because they suffered from respiratory diseases or experienced other serious health problems.

\subsection{Protocol}

From the patients who were treated with conventional periodontal treatment and did not respond well, twenty nine were included in the audit. Each patient had a BPE score and the depth of periodontal pocket from all teeth 
with pockets measured. All had broadly similar levels of disease. The WHO 621 probe was used to measure the depth of pockets. The probe was placed gently in the pocket and the depth was measured in millimetres according to the scale on the probe. BPE scores were measured according to recommendations of British Society of Periodontology [17].

A complete radiographic survey was performed for every patient so as to help in diagnosis. The recruited patients were treated with a combination of conventional periodontal treatment and gaseous ozone application for $18 \mathrm{sec}$ in every pocket (according to the manufacturer's instructions). After the treatment oral hygiene instructions were given to the patients and follow up appointments were scheduled in 6 weeks and in three months time.

Patients were asked to keep their oral hygiene daily dairy. On follow up appointments all the patients had periodontal pockets measured and they were also asked about any problems after ozone treatment. The questions were about the status of the gingiva (bleeding or any discomfort) in the following days after the treatment with ozone. This was in a form of verbal questionnaire during the follow up appointment. On the three months follow up appointment BPE scores were also measured. Treatment with ozone was not repeated on follow up appointments. All the measurements, treatment and follow up appointments were carried out by one of the authors.

The six deepest pockets were taken into account when applying statistical analysis. Differences in pockets depth before and after application of ozone were analysed using the t-test. Statistical analysis was performed with the SPSS software package.

\subsection{Equipment}

Ozotop (TTT, Switzerland) an ozone generator that was available in the author's practice was used (Serial Number: 0307/2006) as shown in Figure 1. This generator uses a corona discharge system and it produces $30 \mathrm{mg}$ ozone per hour. There are three different tips for the oral cavity and the perio-tip was used for the periodontal treatment. The generator has no built-in scavenging system so suction with the special accessory for ozone mouth evacuation (Ozodam, TTT Switzerland) was used, according to manufacturer instructions. Suction with Ozodam was positioned close to the perio-tip in order to minimize the concentration of ozone in the mouth as this approach increases the safety of ozone use [18].

\section{RESULTS}

From the initial number of twenty-nine patients (16 females, 13 males), twenty-five patients attended both follow up appointments ( 6 weeks and 3 months) and four of the patients failed to attend either one or both appoint- ments and consequently were excluded from the analysis. All the patients were asked to keep their oral hygiene daily diary but only sixteen complied with this instruction and returned their diaries.

Only BPE scores and depth of pockets measured on second follow up appointment were taken into consideration and were included in the analysis. The purpose of the first follow up appointment was to make sure that all patients complied with the oral hygiene instructions given and to evaluate the initial results as seen by the difference in the depth of pockets.

The depths of pockets before and after ozone treatment were measured as described in protocol. Based on BPE scores it was apparent that twenty $(80 \%)$ of the patients have improved overall. Fifteen had improvement in more than three regions and five in one or two regions. Five of the patients not only did not improve but had deteriorated. Figure 2 shows the change in BPE scores for each the 25 patients who attended both appointments.

The six deepest pockets were taken into account when applying statistical analysis (before and after the treatment). Figure 3 illustrates the pocket depth measurements of the six deepest pockets before and after ozone application. Eight patients had an improvement in depths of periodontal pockets by three millimetres, sixteen patients had improvement by one to two millimetres and one patient did not improve and the pocket depth had increased by one millimetre. The depth of pockets after the use of ozone decreased significantly $(\mathrm{P}<0.001)$. The measurements were taken three months after the initial ozone treatment.

\section{DISCUSSION}

It has been reported by many authors and supported by this literature review that ozone has an antimicrobicidal effect. Ozone may be effective as it is known to kill micro-organisms by rupturing their cell walls and cytoplasmic membranes [19]. This involves chemical modification and fragmentation of mono unsaturated and polyunsaturated fatty acids in the cell wall [20]. When the membrane is damaged, its permeability increases and ozone molecules can readily enter the cells [19].

It is known that specific bacteria are implicated as causative factors that can lead to the development of periodontitis and that oral microbial plaque consists of different types of bacteria that live on host surfaces. Current treatment of periodontitis is based on disinfecting the supragingival and subgingival tissues in order to re-establish a microorganism-free environment [21]. This fact renders ozone application reasonable and justifiable treatment option [8]. It has been shown in-vitro that ozone is bactericidal against periodontopathic microorganisms [9].

In the present audit gaseous ozone was used. The choice of gaseous ozone was based on the ozone generator cur- 


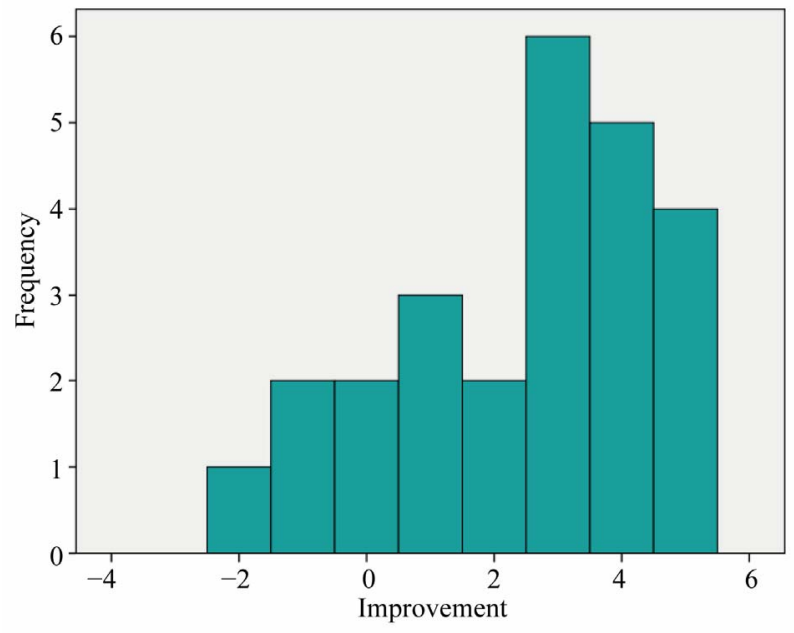

Figure 2. The improvement in BPE scores for each the 25 patients showing changes in score after ozone application, overall showing an improvement $(\mathrm{P}<0.001)$.

BPE Scores Before
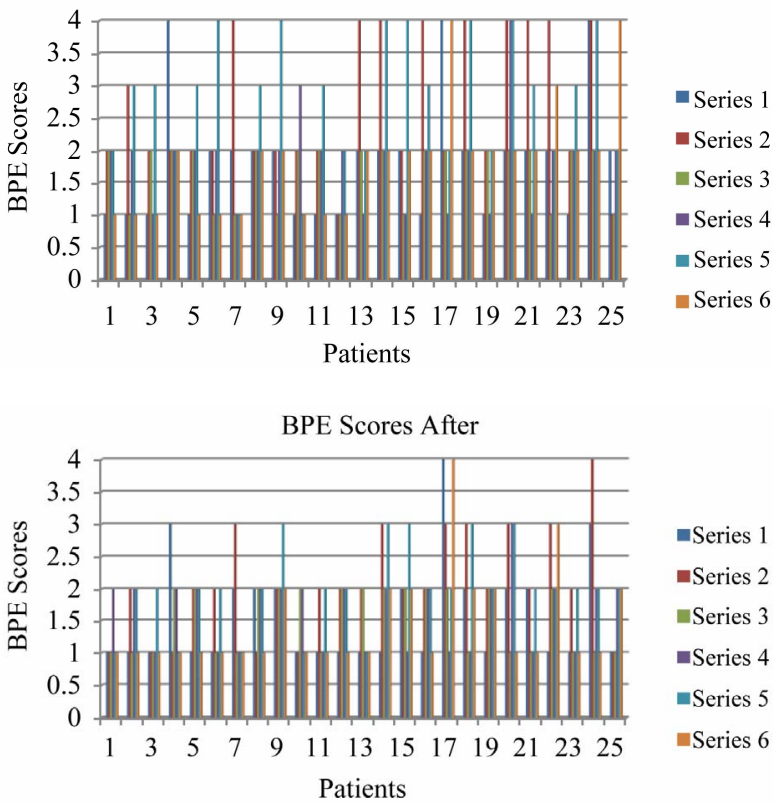

Figure 3. Differences in pockets depth before and after application of ozone for 25 patients included in the audit, series $1-6$ indicated the same six deepest pockets measured in each patient. Overall improvement was significant $(\mathrm{P}<0.001)$.

rently available and in use by the authors, it has been approved for use in the EU and room contamination and staff and patient exposure can be significantly reduced by adherence to safety measures including application of suction $[18,22]$. Attention to safety is important with ozone. In addition, this form of ozone application has not been studied extensively in the periodontal literature. Ozone nano-bubble water has been also studied and shown to have potential as an adjunct to periodontal treatment [10].
To make sure that the observed therapeutic effect was that of ozone patients were also instructed not to use any of the available antimicrobials after the treatment with ozone. It is known that agents like chlorhexidine are being used as part of the chemo-mechanical treatment approach of chronic periodontitis and can significantly reduce the depth of deep and medium-deep pockets [22]. Patients were given oral hygiene instructions and were asked to keep their oral hygiene daily diary.

In the present audit, the patients displayed a variety of teeth with periodontal disease including localization, number of diseased teeth and variability of surfaces involved. It was decided that only the six deepest pockets from every patient will be included in the audit. These pockets were only measured before and after the application of gaseous ozone. This approach although practical might have affected the BPE score. In some patients, although all the teeth with pockets including the six deepest improved by few millimetres, their BPE score did not. This may be due to the fact that for various reasons not all the treated pockets improved. It might be that these were the patients who did not keep systematically daily diaries and consequently did not comply with the given oral hygiene instructions.

The present audit showed an overall beneficial effect of ozone application with $80 \%$ of the patients showing significantly $(\mathrm{P}<0.001)$ improved BPE scores after gaseous ozone application. Additionally, pocket depth analysis revealed that $32 \%$ of the deepest pockets improved by more than three millimetres, $64 \%$ by one to two millimetres and $4 \%$ did not improve or deteriorated. The results support the use of ozone as an adjunct to mechanical treatment in periodontal patients in agreement with other studies $[9,10]$.

However, there are recognised limitations to the present audit as it was not a research study and so no control group was possible. First, this is not a randomized, controlled and blinded study with all the limitations that this may have and inevitably there may be observational bias introduced, as all the measurements were taken by the author. One another factor that may also affect the validity and reliability of the obtained results is the small number of patients included in the audit. This may introduce type II error. In addition, the statistically significant results found in the audit may not translate necessarily into clinically significant results. One additional factor is the short length of follow-up period in this study. It is important to obtain long lasting therapeutic results in order to justify the use of ozone. In this audit the aspect of cost effectiveness was not addressed although it is an additional and important factor in clinical practice and the positive results in long term should justify the cost of ozone therapy.

The patients included in the audit did not experienced 
respiratory or other medical problems and there were no adverse effects observed after the use of ozone. However, they were not screened for smoking although the deleterious effects of smoking on periodontal health have been well-documented [23-25]. This might be responsible for some patients not responding to the treatment. However, the patient population represented subjects who did not respond to conventional periodontal treatment. It is possible that patients who are diagnosed and treated for the first time might have responded more favourably to ozone treatment. Nevertheless, the authors wished to audit the usefulness of ozone in more resistant cases.

\section{CLINICAL SIGNIFICANCE}

Ozone offers a simple adjunctive therapy for managing periodontal disease alongside convention methods. Further research is indicated.

\section{CONCLUSION}

This audit showed beneficial results of gaseous ozone treatment in patients with periodontal disease. These are encouraging results that call for further future randomised controlled studies to obtain high quality evidence for clinical practice.

\section{REFERENCES}

[1] Lynch, E. (2004) Ozone: The revolution in dentistry. Quintessence Publishing Co Ltd., Surrey.

[2] Rubin, M.B. (2001) The history of ozone. The Schonbein period, 1839-1868. Bulletin Histology and Chemistry, 26, 40-56.

[3] United States Environmental Protection Agency (1999) Alternative disinfectants and oxidants. EPA, 815-R-99014.

[4] Azarpazhooh, A. and Limeback H. (2008) The application of ozone in dentistry: A systematic review of literature. Journal of Dentistry, 36, 104-116. doi:10.1016/j.jdent.2007.11.008

[5] Saini, R. (2011) Ozone therapy in dentistry: A strategic review. Journal of Natural Science Biology and Medicine, 2, 151-153. doi:10.4103/0976-9668.92318

[6] Hodson, N. and Dunne, S.M. (2007) Using ozone to treat dental caries. Journal of Esthetic and Restorative Dentistry, 19, 303-305. doi:10.1111/j.1708-8240.2007.00127.x

[7] Skurska, A., Pietruska, M.D., Paniczko-Drężek, A., Dolińska, E., Zelazowska-Rutkowska, B., Zak, J., Pietruski, J., Milewski, R. and Wysocka, J. (2010) Evaluation of the influence of ozonotherapy on the clinical parameters and MMP levels in patients with chronic and aggressive periodontitis. Advances in Medical Science, 55, 297-307. doi:10.2478/v10039-010-0048-x

[8] Gupta, G. and Mansi, B. (2012) Ozone therapy in perio- dontics. Journal of Medical Life, 22, 59-67.

[9] Eick, S., Tigan, M. and Sculean, A. (2012) Effect of ozone on periodontopathogenic species - An in vitro study. Clinical Oral Investigations, 16, 537-544. doi:10.1007/s00784-011-0515-1

[10] Hayakumo, S., Arakawa, S., Mano, Y. and Izumi, Y. (2013) Clinical and microbiological effects of ozone nanobubble water irrigation as an adjunct to mechanical subgingival debridement in periodontitis patients in a randomized controlled trial. Clinical Oral Investigations, 17, 379-388.

[11] Walker, J.T., Dickinson, J., Sutton, J.M., Raven, N.D.H. and Marsh, P.D. (2007) Cleanability of dental instruments-Implications of residual protein and risks from Creutzfeldt-Jacob disease. British Dental Journal, 203, 395-401. doi:10.1038/bdj.2007.893

[12] Bezirtzoglou, E., Cretoiu, S.M., Moldoveanou, M., Alexopoulos, A., Lazar, V. and Nakou, M. (2008) A quantitative approach to the effectiveness of ozone against microbiota organisms colonizing toothbrushes. Journal of Dentistry, 36, 600-605. doi:10.1016/j.jdent.2008.04.007

[13] Baysan, A. and Lynch, E. (2005) The use of ozone in dentistry and medicine. Primary Dental Care, 12, 47-52. doi:10.1308/1355761053695158

[14] British Society of Periodontology (2001) Periodontology in general dental practice in the United Kingdom. A policy statement. British Society of Periodontology, 1-12.

[15] Kshitish, D. and Laxman, V.K. (2010). The use of ozonated water and $0.2 \%$ chlorhexidine in the treatment of periodontitis patients: A clinical and microbiological study. Indian Journal of Dental Research, 21, 341-348. doi:10.4103/0970-9290.70796

[16] Hauser-Gerspach, I., Vadaszan, J., Deronjic, I., Gass, C., Meyer, J., et al. (2011) Influence of gaseous ozone in periimplantitis: Bactericidal efficacy and cellular response. An in vitro study using titanium and zirconia. Clinical Oral Investigations, 16, 1049-1059. doi:10.1007/s00784-011-0603-2

[17] Huth, K.C., Quirling, M., Lenzke, S., Paschos, E., Kamereck, K., Brand, K., Hickel, R. and Ilie, N. (2011) Effectiveness of ozone against periodontal pathogenic microorganisms. European Journal of Oral Science, 119, 204 210. doi:10.1111/j.1600-0722.2011.00825.x

[18] Millar, B.J. and Hodson, N. (2007) Assessment of the safety of two ozone delivery devices. Journal of Dentistry, 35, 195-200. doi:10.1016/j.jdent.2006.07.010

[19] Nagayoshi, M., Fukuizumi, T., Kitamura, C., Yano, J., Terashita, M. and Nishihara, T. (2004) Efficacy of ozone on survival and permeability of oral micro organisms. Oral Microbiology and Immunology, 19, 240-246. doi:10.1111/j.1399-302X.2004.00146.x

[20] Grootveld, M., Baysan, A., Siddiqui, N., Sim, J., Silwood, C. and Lynch, E. (2004) Ozone: The revolution in dentistry. Quintessence Publishing Co. Ltd., Surrey, 23-30.

[21] Cosyn, J., Wyn, I., De Rouck, T., et al. (2007) Subgingival chlorhexidine varnish administration as an adjunct to same-day full-mouth root planning. I. Clinical observations. Journal of Periodontology, 78, 430-437. 
doi:10.1902/jop.2007.060221

[22] Johansson, E., Anderson-Wenckert, I., Hagenbjork-Gustafson, A. and Dijken, J.W.V. (2007) Ozone air levels to a dental ozone gas delivery system. Acta Odontologica Scandinavica, 65, 324-330. doi:10.1080/00016350701687247

[23] Cronin, J.A., Claffey, N. and Stassen, L.F. (2008) Who is at risk? Periodontal disease risk analysis made accessible for the general dental practitioner. British Dental Journal, 205, 131-137. doi:10.1038/sj.bdj.2008.653
[24] Feldman, R.S., Bravacos, J.S. and Rose, C.L. (1983) Association between smoking different tobacco products and periodontal disease indexes. Journal of Periodontology, 54, 481-487. doi:10.1902/jop.1983.54.8.481

[25] Grossi, S.M., Zambon, J.J., Ho, A.W., Koch, G., Dunford, R.G., Machtei, E.E., et al. (1994) Assessment of risk for periodontal disease. I. Risk indicators for attachment loss. Journal of Periodontology, 65, 260-267. doi:10.1902/jop.1994.65.3.260 